Der Wedekind-Preis für deutsche Geschichte 2009 wurde Herrn Horst Walter Blanke, Bielefeld, für die Edition zu Johann Gustav Droysens „Historik" verliehen.

\title{
Die Historik J. G. Droysens. Ein Editionsprojekt
}

\section{Horst Walter Blanke}

Bei der Fortsetzung der historisch-kritischen Edition der Droysenschen Historik handelt(e) es sich um ein von der DFG gefördertes Projekt, das insgesamt fünf Jahre (Mai 2004 bis April 2009) dauerte, das aber noch nicht abgeschlossen ist.

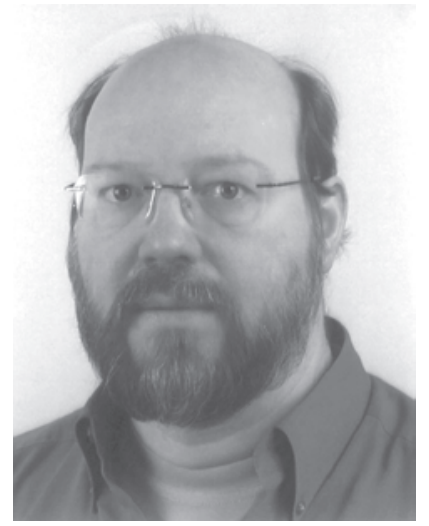

Horst Walter Blanke, Professor für Neuere Geschichte an der Universität Bielefeld, Träger des Wedekind-Preises 2009

\section{Ausgangspunkt}

Bevor ich auf Droysens Historik zu sprechen komme, seien einige Bemerkungen zur Historik generell gestattet: „Historik“ stellt ein Moment der Verfachlichung und der Fachlichkeit der Geschichtswissenschaft selbst dar, indem sie die Praxis der verwissenschaftlichten Geschichtsschreibung und der methodisch geregelten historischen Forschung bestimmt. Damit nimmt sie fünf verschiedene Funktionen wahr: Sie dient (1) didaktisch-propädeutischen Zwecken, (2) der Systematisierung historischen Wissens, (3) der Spezialisierung in bestimmte Forschungsmethoden und Arbeitsgebiete, (4) v. a. der systematischen Begründung von Eigenart und Funktion der Geschichtswissenschaft im Zusammenhang mit anderen Wissenschaften und im lebenspraktischen Kontext der Historiker und ihres Publikums und (5) der historischen Absicherung erreichter Standards der Wissenschaftsentwicklung, letzteres v. a. in der Form der Historiographiegeschichte.

Droysens Historik hat zahlreiche Wandlungen und Editionen durchgemacht (s.u. Abschnitt 5); 1977 erschien ein erster Band (von drei geplanten) der kritischen Ausgabe, der von Peter Leyh veranstaltet wurde: ein gewaltiger Torso, der geradezu darauf wartet(e), fortgesetzt zu werden. Der DFG sind zwei Publikationen versprochen: die Texte aus dem Umkreis der Historik und die Historik letzter Hand; der erstgenannte Band liegt vor (2007), den 
anderen hoffe ich Ende dieses Jahres druckfertig zu haben, so daß er im Sommer 2012 erschienen sein wird.

Allerdings: Neben diversen Aufsätzen sind zwei weitere Publikationen über Droysen - gewissermaßen als Geschenk an die DFG - erschienen: eine umfassende Droysen-Bibliographie (2008) und eine Festschrift zum 70. Geburtstag Jörn Rüsens, die, erheblich erweitert, aus den Tagungsbeiträgen zum 200. Geburtstag Droysens hervorgegangen ist, eine Festschrift, die Rüsens Schriftenverzeichnis und ansonsten ausschließlich Beiträge über Droysen enthält (2009).

Eine preiswerte, auch für Studenten erschwingliche Studienausgabe der Droysenschen Historik soll etwa drei Jahre nach Erscheinen des dritten Bandes, der Historik letzter Hand, publiziert vorliegen [1].

So ist als ein Zwischenfazit festzuhalten: die versprochenen Resultate liegen zwar noch nicht vollständig vor, werden aber ergänzt um weit über sie hinausgehende Publikationen.

Wofür habe ich den Preis bekommen? - Die Urkunde vom 21. November 2009 sagt folgendes aus: „Für die Edition zu Johann Gustav Droysens ,Historik“ “. Da der dritte Band der historisch kritischen Edition noch nicht fertig ist, bleiben meine Ausführungen auf die zwei bzw. drei erschienenen Bände beschränkt.

\section{Droysen-Bibliographie}

Die Bibliographie ist der Versuch einer möglichst vollständigen und bibliographisch korrekten Auflistung der Schriften Droysens, ferner der Verzeichnisse der Teilnachlässe in Berlin-Dahlem, in Jena und in Halle (Saale). Darüber hinaus erfolgte der Abdruck eines zwar bedeutsamen, aber dennoch fragmentarischen persönlichen Schriftenverzeichnisses Droysens, des sog. Katalogs meiner Arbeiten. Bedeutsam ist dieser Katalog insofern, als er einerseits chronologische und sachliche Erläuterungen zu einzelnen Publikationen liefert [2], andererseits auch sog. graue Literatur zitiert [3].

\section{Die Entstehung der Droysenschen Historik}

Die Entstehung und Herausbildung der Droysenschen Historik aus unterschiedlichen thematischen Zusammenhängen und aus verschiedenen Textsorten ist eine spannende Frage. Droysen hat im Jahr 1852 erstmals eine Historik-Vorlesung angekündigt, aber erst fünf Jahre später tatsächlich gelesen. Die Gründe für diese Verzögerung sind nicht bekannt. 1857 entstand 
ein stichwortartiger Grundriß der Historik; die diesbezüglichen Vorlesungstexte liegen aus dem Nachlaß ediert vor (1977). In der Fassung des Grundrisses vom Jahre 1868 finden sich als Beilage drei theoretische Aufsätze, nämlich Die Erhebung der Geschichte zum Rang einer Wissenschaft (1863), eine Auseinandersetzung mit dem Positivismus, ferner Natur und Geschichte (1866) sowie Kunst und Methode (1867), die beide zentrale Punkte der Droysenschen Historik behandeln. Scheinbar sind alle bedeutenden Texte zur Historik erst 1857 und später entstanden. Indes, der Schein trügt. Denn natürlich beruht die Historik Droysens nicht auf einer genialen Eingebung, sondern speist sich aus verschiedenen Traditionssträngen. Diese sind in der Sammlung Texte im Umkreis der Historik, dem 2. Band der historisch-kritischen Ausgabe, zusammengestellt worden [4].

\section{Texte im Umkreis der Historik}

Der genannte Band enthält 50 Texte, von denen 40 bis zum Jahre 1857, das gewissermaßen als Stichdatum dient, entstanden sind. (Denn Texte der Jahre $1858 \mathrm{ff}$. sind nur insoweit berücksichtigt, als sie theoretische Themen behandeln, die im Rahmen der Historik-Vorlesung nicht oder doch nicht ausführlich behandelt worden sind, etwa Fragen des Wissenschaftsbetriebes wie z. B. der der Organisation des Geschichtsstudiums.)

Die verschiedenen Traditionsstränge korrespondieren auch unterschiedlichen Textgattungen: (1) Vorlesungseinleitungen, (2) Briefen, (3) Buchbesprechungen, (4) Denkschriften, (5) Aufsätzen, (6) Vorträgen, auch (7) Gedichten sowie schließlich (8) Akten. Daneben ist als eine eigenständige Textsorte die sogenannte Privatvorrede in dem 2. Band der Geschichte des Hellenismus zu nennen, ein Text, der gelegentlich auch unter dem Titel Theologie der Geschichte publiziert worden ist. Mit 20 Nummern bilden zweifellos die Vorlesungseinleitungen die zahlenmäßig größte Gruppe; diese Einleitungen bilden nicht selten so etwas wie Mini-Historiken bzw., treffender, „Proto-Historiken“.

Welchen Beitrag zur Theorie-Diskussion leisten nun diese Texte? Nun, der Beitrag ist durchaus unterschiedlich.

Der erste von fünf Hauptteilen, der Texte aus Droysens Berliner Studentenzeit umfaßt (1826-31), enthält strenggenommen keine Texte zur Historik, wohl aber eindrucksvolle Dokumente zur Entwicklungsgeschichte der Droysenschen Denkens: In einem Gedicht aus dem Jahre 1827 z. B. wird "Geschichte" als Widerspiegelung des Göttlichen gefeiert; in den Briefen an die Schwestern wird Droysens tiefe Religiosität deutlich, die auch in dem Aufsatz Ueber die Passions-Musik von J. S. Bach artikuliert wird, hier 
freilich mit der Variante, den Staat Preußen als die Inkarnation des Protestantismus erweisen zu wollen. Die Gedichte dokumentieren Droysens Vertrautheit mit den Mythen der klassischen Antike. Nicht von ungefähr hat Droysen in seinem handschriftlichen Schriftenverzeichnis Katalog meiner Arbeiten selbstironisch diesen Abschnitt seines literarischen Schaffens mit den Worten kommentiert: „Wie natürlich spielten am Anfang poetische Versuche eine nicht geringe Rolle“.

Der zweite Hauptteil dokumentiert die Berliner Zeit als Privatdozent und apl. Professor, d. h. die Jahre 1833-38. Die Antrittsrede zum Privatdozenten formuliert als Hauptaufgabe einer Beschäftigung mit dem griechischen Drama, der Tragödie und auch der Komödie, diese in ihrem historischen Werden darzustellen. In der Auseinandersetzung zwischen Sach- und Sprachphilologie bezog Droysen eindeutig Position für die Realphilologie.

In den Buchbesprechungen erfolgte eine Auseinandersetzung mit der Historiographiegeschichte, mit den großen Vorbildern: Barthold Georg Niebuhr galt als Wegbereiter der modernen Geschichtswissenschaft, ebenso Leopold Ranke.

In den Beschreibungen und Reflexionen zur Berliner Kunstausstellung 1834 kam es zu einer Beschäftigung mit der Historienmalerei: in Einzelkritiken werden die strukturellen Grenzen der Historienmalerei aufgezeigt, die u. a. darin bestünden, daß das, was den geschichtliche Moment - z. B. einen Wendepunkt in der geschichtlichen Entwicklung - auszeichnet, oft nicht überzeugend bildlich vermittelt werden könne. Daneben, eher beiläufig, kam Droysen zu Aussagen wie der folgenden: die abstrakte Wahrheit sei ebenso unwahr wie die porträtierende Naturwahrheit. Es gelte also, ein Mittelmaß zwischen Abstraktion und Empirie zu finden.

In den Briefen an Freunde war Droysen etwas frecher. Die Historiker seien oft nicht imstande (dieses „oft“ fehlt freilich im Original), die Notwendigkeit des Geschehenen zu begreifen. Oder es werden geschichtsphilosophische Überzeugungen formuliert. Um wenigstens ein Beispiel zu nennen: der Geschichte des einzelnen Menschen ist die der Menschheit analog.

Am Beispiel der Einleitung in die Vorlesung zur Alten Geschichte kann man die intellektuelle Entwicklung Droysens verfolgen: 1838 ist der Abriß nur kurz; in sechs Abschnitten (auf 51/2 Seiten) werden folgende Themen kurz behandelt: Was ist Geschichte? Wo beginnt die Geschichte? Was ist die alte Geschichte? Quellen? Hülfswissenschaften (Geographie, Chronologie, auch: Staatswissenschaften, schließlich: Kriegswissenschaften)? „Hülfsbücher" (= Gesamtdarstellungen, Forschungsliteratur)? [5]

Der dritte Hauptteil umfaßt die Kieler Zeit, d. h. die Jahre 1840-47. In meiner Sammlung sind sie durch insgesamt 17 Texte dokumentiert, davon 
13 Vorlesungseinleitungen. In dem von Droysen und Waitz entworfenen Kieler Curriculum des Geschichtsstudiums werden eine „Encyclopädie der Geschichte“ sowie eine „Philosophie der Geschichte“ als Nebenvorlesungen angeregt. Ferner hat Droysen ein Gutachten über die Entwicklung des Gelehrtenschulwesens (Gymnasium) verfaßt: hier wird u. a. die Verortung der Geschichte im Lehrplan erörtert und wird - neben anderen Punkten die Forderung nach Einrichtung eines Pädagogischen Seminars erhoben. In der sog. Privatvorrede finden wir eine Auseinandersetzung und Erläuterung des Hellenismusbegriffes. Als Ziel der Geschichte wird hier ganz unbefangen die Theodizee formuliert und als Forderung die Notwendigkeit einer Historik artikuliert!

Nun aber zu den Vorlesungseinleitungen, die eine große thematische Vielfalt bergen [6]. Droysen hat Vorlesungen gehalten über sämtliche Großepochen: die Alte Geschichte, die Geschichte des Mittelalters, die Neuere Geschichte, die Neueste Geschichte (seit 1815, also die „Zeitgeschichte"); daneben hat er sich mit thematischen Zuspitzungen beschäftigt, nämlich mit der deutschen Geistesgeschichte, der Geschichte der Freiheitskriege, der Geschichte des Deutschen Bundes, der Geschichte der Revolutionen und schließlich mit den bereits erwähnten Politikvorlesungen (Ueber den öffentlichen Zustand Deutschlands).

Zunächst eine Vorbemerkung: Der Gehalt dieser 13 Vorlesungseinleitungen erschöpft sich nicht darin, Vorläufer der Historik-Vorlesung zu sein. (Immerhin ist dies als Forderung explizit formuliert.) Der Gehalt erstreckt sich auf drei verschiedene Ebenen: (1) Der Geschichtsverlauf wird auf den Punkt gebracht: er zeigt immer wieder aufs Neue die Auseinandersetzung über das, was Geschichte als Sachverhalt auszeichnet, meist als Vorgeschichte der jeweiligen Vorlesung. Diese Passagen enthalten z.T. bereits geschichtsphilosophische Passagen bzw. Bemerkungen. So werden z.B. die Entitäten Monarchie und Staat als historische Kategorien reflektiert; es wird ein dreigliedriges Kategorienraster der Geschichte: Politik - Gesellschaft - Kultur entwickelt. Als höchste Aufgabe der Geschichtswissenschaft - das haben wir bereits kennengelernt - gilt die Theodizee.

Diese geschichtsphilosophischen Ausführungen finden sich am ehesten in der „Systematik“ der Historik wieder. Sie lassen sich erweitern: Als Aufgabe der Geschichte wird der geistige Inhalt herausgearbeitet, den das Leben und die Entwicklung der Menschheit habe. Der Gehalt der Vorlesungseinleitungen erstreckt sich ferner (2) auch auf Bestandteile der „Methodik“, so etwa in der These, daß der Historiker nie ohne Parteiinteressen schreiben könne. 
Am Beispiel der Alten Geschichte läßt sich der letzte, der dritte Punkt veranschaulichen: der Erkenntnisfortschritt ist unverkennbar. Zwar wird die alte Sechserteilung beibehalten, aber sie wird nun inhaltlich neu gefüllt, und - vor allem - sie wird reflektiert: Der Abschnitt Wo beginnt Alte Geschichte?, um nur ihn zu nennen, unterstreicht nun die Eigentümlichkeiten der Sprache, der gesellschaftlichen Strukturen, der Religion; die Differenzierungen der Ausführungen über die "Quellen“ dokumentieren den Erkenntniszuwachs. Schließlich: Der Umfang hat sich vervierfacht: Aus 5 $\frac{1}{2}$ Seiten sind 221/2 Seiten geworden [7].

Der vierte Hauptteil umfaßt die Jenaer Jahre 1850-58 mit insgesamt neun Texten: die Jenaer Antrittsvorlesung, persönliche Briefe, Vorlesungseinleitungen und schließlich eine Erläuterung von Eduard Bendemanns Wandgemälden im Dresdner Schloß. Letztere bildet in gewisser Weise ein Pendant zu dem Bericht über die Berliner Kunstausstellung (s. o.).

Die Antrittsvorrede thematisiert u. a. die enge Verwandtschaft von Geschichte und Politik, ferner die Abgrenzung der Geschichte von der Philosophie und der Theologie und kulminiert schließlich in der pathetischen Aussage: „Der Gattungsbegriff der Menschen ist die Geschichte“.

In den Briefen wird Historik einerseits als Waffe gegen den Materialismus begründet, andererseits gegen die kritische Schule Rankes - ganz im Gegensatz zur Lobhudelei der 1830er Jahre (s.o.). Selbstbewußt betont Droysen seine Pionierrolle - „zum ersten Male“-, begründet er z. B. seine Ausführungen über vier verschiedene historische Darstellungsformen.

Was die Vorlesungseinleitungen betrifft, so setzen sich die oben beobachteten Trends fort. Zugleich kommt es zu einer Verschiebung der Argumentation: Die Zweckbestimmung des Staates (Politikvorlesung) ist Macht eine solche Sichtweise war wohl erst nach den Erfahrungen der gescheiterten 1848/49er Revolution möglich. Geschichte als Zeugnis der göttlichen Weltregierung - das ist in den 1850er Jahren eine eher seltene Formulierung, häufiger wird die Gegenwart als Krisenerfahrung bestimmt.

Besonders deutlich werden die Änderungen in den Vorlesungseinleitungen über die Alte Geschichte. Diese werden erneut erweitert. 1857 gibt es eine klare Abgrenzung von den Naturwissenschaften, es finden sich Auseinandersetzungen mit Francis Bacon und mit Aristoteles. Als zentrales Moment der geschichtswissenschaftlichen Methode wird das „forschende Verstehen", die Interpretation, hervorgehoben [8].

Der fünfte Hauptteil, der insgesamt zehn Texte eines Vierteljahrhunderts umfaßt, berücksichtigt v. a. Einzelaspekte der Historik. Er reproduziert sechs Denkschriften über die Organisation des Geschichtsstudiums oder die Ausgestaltung des Archivwesens. Diese Gutachten waren nur zum 
Teil bestellt; zum Teil hat Droysen sie unaufgefordert verfaßt, um politischen Einfluß zu nehmen. Diese Liste wäre auch zu erweitern.

\section{Die weitere Entwicklung der Droysenschen Historik und ihre Edition}

Auf die weitere Entwicklung der Droysenschen Historik kann und will ich hier nicht detailliert eingehen; nur soviel: Droysen hat die HistorikVorlesung in dem Vierteljahrhundert bis zum Wintersemester 1882/83 einschließlich weitere 16mal gehalten; dabei hat er den Text fortwährend überarbeitet, d.h. gekürzt, erweitert, umgeschrieben, umgestellt.

Es ist allerdings nicht möglich, eine jeweils vollständige Version aller 17 Fassungen zu rekonstruieren. (Etwa in Form einer CD-Rom-Edition. Es gibt zu viele Löcher, zu viele Ungenauigkeiten. Außerdem: der Arbeitsaufwand wäre m. E. zu groß. Außerdem gibt es ein Kardinalproblem: die Streichungen.) Schon Peter Leyh hat sich für seine Rekonstruktion der ersten vollständigen Fassung der Vorlesungen (1857) auf alle Überlieferungsschichten, auch die der späten, bis hin zur Version von 1882/83, gestützt. Manche Textpassagen können einzelnen Überlieferungslagen nur vage zugeschrieben werden, so daß oft keine Zuordnung auf ein einzelnes Semester erfolgen kann.

Das ist auch ein gewichtiger Grund, weshalb als 3. Band der historischkritischen Ausgabe der Historik „die Historik letzter Hand“ rekonstruiert werden soll (d.h. zur Zeit von mir rekonstruiert wird): die Gegenüberstellung von einerseits der frühesten (wenigstens z.T.) und andererseits der spätesten Fassung kann und muß als Dokumentation der Entwicklungsgeschichte der Droysenschen Historik weitgehend genügen.

\section{Die Aufgabe des Editors}

Die Aufgabe des Editors sind vielfältiger Art. Zunächst geht es darum, aus den handschriftlichen Befunden einen Text zu rekonstruieren, der der in einem bestimmten Semester tatsächlich gehaltenen Vorlesung möglichst nahekommt. Streichungen und Neuformulierungen sind zu berücksichtigen. Nicht selten sind die Reihenfolge und die Zuordnung zu einer bestimmten Signatur defekt, sie müssen dann in ihre richtige Ordnung gebracht werden. (Eine Hilfe sind Droysens Markierungen auf dem Rand, die anzeigen, wie weit er mit dem Stoff gekommen ist.) Zusätze und Verbesserungen (auch orthographischer Art) des Editors werden durch entsprechende Siglen (etwa: eckige Klammern) deutlich gemacht. Die Seitenzählung 
der Originale werden als Marginalien angezeigt [9]. Schließlich: Wo es keine autographische Überlieferung mehr gibt (bzw. diese bislang unauffindbar waren), sind die apographischen Befunde heranzuziehen und entsprechend deutlich zu machen [10].

Der Editor rekonstruiert so einen lesbaren und möglichst vollständigen Text, der gleichwohl durch diakritische Symbole, Fußnoten und Hinweise auf die "kommentierenden Anmerkungen“ seiner Lesbarkeit ein wenig abträglich ist [11]. Meines Erachtens gibt es dazu keine Alternative. Die „kommentierenden Anmerkungen“ berichten über Textbefunde und lösen bibliographische Anspielungen auf, erläutern wichtige Sachverhalte, ohne deren Kenntnis die Droysenschen Texte nur schwierig zu verstehen sind [12].

Schließlich ist ein editorischer Bericht zu schreiben, der die Eingriffe und Befunde darlegt.

Im Falle des 2. Bandes der Historik Droysens sind über die oben angeführten Arbeitsschritten hinaus noch essentielle Fragen gestellt worden: nach der Anzahl der Texte, die z.T. direkt und auch indirekt erfolgten. Weitere Texte sind u. U. sinnvoll, aber m. E. nicht zwingend [13]. Ich bin sicher, die wichtigsten Texte versammelt zu haben, die den Entwicklungsprozeß Droysen dokumentierten. (Die Jenaer Antrittsrede wurde auf lateinisch gehalten - eine deutschsprachige bzw. deshalb bilinguale Fassung dient letztlich der Verständlichkeit [14].) Die einzelnen Texte unterliegen nicht einer chronologischen, sondern einer thematischen Anordnung; sie ergänzen sich gegenseitig.

\section{Literatur}

[1] (a) J. G. Droysen: Hist.-krit. Ausgabe, Bd.1: Rekonstruktion der ersten vollständigen Fassung der Vorlesungen (1857), Grundriß der Historik in der ersten handschriftlichen (1857/1858) und in der letzten gedruckten Fassung (1882), hg. v. P. Leyh, Stuttgart 1977; (b) J. G. Droysen: Historik. ..., Bd. 2: Texte im Umkreis der Historik (1826-84), hg. v. H. W. Blanke, Stuttgart 2007; (c) J. G. Droysen: Historik ... Supplement: Droysen-Bibliographie, hg. v. H. W. Blanke, Stuttgart 2008; (d) H. W. Blanke (Hg.): Historie und Historik. 200 Jahre J.G. Droysen. Festschrift für J. Rüsen zum 70. Geburtstag, Köln u.a. 2009; (e) J. G. Droysen: Historik ... , Bd. 3: Die Historik letzter Hand, hg. v. H. W. Blanke, i. V. (ca. 2012);

(f) J. G. Droysen: Historik, Studienausgabe, hg. v. H. W. Blanke, i. V. (ca. 2015).

[2] Droysen-Bibl., 175 (Nr. V.5).

[3] Ebd., 174 (Nr. IV.11).

[4] Bibl. Nachweise in: Blanke 2009, 28ff.

[5] Historik 2,1, 96-103 (Nr. 14). 
[6] F. Jaeger in Blanke 2009, 106ff über die Einleitungen der Neuzeit-Vorlesungen.

[7] Historik 2,1, 144-67 (Nr. 18).

[8] Historik 2,2, 352-73 (Nr. 35).

[9] Z. B. Historik 2,1, 225-45 (Nr. 25).

[10] Für die in Arbeit befindliche Historik 3 s. Droysen-Bibl., 137 (Nrn. 171 u. 172).

Vgl. etwa Historik 1, 46,11-15 u. 47,36 sowie 243,16-27.

[11] Vgl. Historik 1, 3-6 (mit 491).

[12] Historik 2,2, 559-689.

[13] Etwa Blanke 2009, 33 Anm. 41.

[14] Historik 2,2, 334-51. 